\title{
REGRESSION-BASED MODEL FOR THE EVALUATION OF CYP2D6- MEDIATED DRUG-DRUG INTERACTIONS
}

Lozano R*, Frutos A, Apesteguía A, Martinez A, Franco ME and Alcazar MA, Correspondence to:

* Dr Roberto Lozano. Hospital Real Nuestra Señora de Gracia, Pharmacy Department. Ramon y Cajal 60. 5000 Zaragoza (Spain); Telefono: +34976444300 Fax:

+34876764555 e-mail: rlozano@salud.aragon.es

\section{ABSTRACT}

We aimed to construct a linear regression-based model between area-under-curve (AUCr) and the therapeutic range-to-inhibition constant ratio (TR-to- $\mathrm{K}_{\mathrm{i}}$ ). As result, a linear log-log regression model, between the averaged AUCr, calculated as the mean from the values of different DDIs between several victim-drugs with the same inhibitor $\left(\mathrm{AUCr}_{\mathrm{avg}}\right)$, and the mean TR-to- $\mathrm{K}_{\mathrm{i}}$ ratio $\left(\mathrm{TR}_{\mathrm{m}}-\right.$ to- $\left.\mathrm{K}_{\mathrm{i}}\right)$, calculated as the mean value between the maximum and minimum TR of the inhibitor divided by its $\mathrm{K}_{\mathrm{i}}$, obtained the best correlation $\left(\mathrm{r}^{2}=0.72 ; \mathrm{p}=0.0116\right)$. Accordingly, a drug-drug interaction involving reversible inhibitory drugs of CYP2D6 could be manage by adjusting dose of victim and/or inhibitory drug to the magnitude of the desired change, by applying data of TR and $\mathrm{K}_{\mathrm{i}}$ of inhibitor to the equation of regression line presented here.

\section{KEY WORDS}

Area under curve, inhibition constant, CYP2D6, drug drug interactions, therapeutic range.

\section{INTRODUCTION}

Drug-drug interactions (DDI) are changes in a drug's effects due to the recent or concurrent use of one or more drugs that may become critical to a patient's health. In 
particular, those cytochrome P450 (CYP)-mediated can lead to serious adverse drug reactions (ADR) and constitute one of the main concerns in clinical pharmacology. ${ }^{1}$ However, these clinically relevant DDIs are generally preventable, and their identification, quantification and management should be considered as a main objective in the safe practice of pharmacotherapy.

The Food and Drug Administration, based on equation for basic models of reversible inhibition of CYP enzymes other than CYP3A ( R1=1+[I] $]_{\max } / \mathrm{K}_{\mathrm{i}}$; Eq.1), where R1 is the predicted ratio of the victim drug's area under curve (AUCr) in the presence and absence of an inhibitor, $[\mathrm{I}]_{\max }$ is the maximal unbound plasma concentration of the inhibitor, and $\mathrm{K}_{\mathrm{i}}$ is the unbound inhibition constant (determined in vitro), recommends conducting clinical studies of a given DDI when AUCr at steadystate (SS) of a probe drug (e.g. dextromethorphan), whose clearance is determined by metabolism through the P450 enzyme, is $>1.1 .^{2}$

Successful integration of in vitro to in vivo data is dependent on the inhibitory concentration ([I]) used (e.g., $[\mathrm{I}]_{\text {avg }}$ average plasma concentration, $[\mathrm{I}]_{\max }$ maximal unbound plasma concentration, $[\mathrm{I}]_{\mathrm{h}}$ maximal hepatic concentration), remaining problematic which of they or other should be used in prediction models.

In this way, we know that in clinical pharmacology obtaining plasma concentration of a drug (e.g. $[\mathrm{I}]_{\max }$ ) is only readily available for a small number of drugs with low therapeutic index (e.g., digoxin, immunosuppressants).Thus, the use of therapeutic range (TR) data, since it refers both to the dosage range and to the plasma concentration at which the desired therapeutic effect is obtained, and due to its easy accessibility, could be used as a substitute for $[\mathrm{I}]_{\max }$ in Eq. 1. Also, for an assessment of DDI effects in vivo, along with [I], $\mathrm{K}_{\mathrm{i}}$ values are required, which are dependent upon inhibitor and substrate but independent of substrate concentration used. 
On the other hand, depending on the mode of interaction between CYP enzyme and inhibitor, reversible CYP inhibition, that occur in an inhibitor dose (concentration)dependent manner in the clinical setting, may be further described as competitive. ${ }^{3}$ When this competitive inhibition occurs, the IC50, a parameter used for Ki-calculation by means of the Cheng-Prusoff equation, ${ }^{4}$ rises and, thus, the degree of inhibition reduces along with the increase in the concentration of the victim-drug. ${ }^{5}$

Because of this, our objective has been to correlate the variations in interaction intensity (AUCr) with those of $[\mathrm{I}]_{\max }$, for a set of known inhibitors of the CYP2D6, aimed to evaluate the clinical evaluation of CYP2D6-mediated DDIs.

\section{MATERIAL AND METHODS}

To choose between multiple possible correlations, we previously carried out a theoretical approach based on the fact that Eq.1 is fulfilled at all points of the regression line and, on the other hand, that between one point (point [1]) and the next (point [1']) of the regression-line, there is a change of $\mathrm{AUCr}$ and $[\mathrm{I}]_{\max }$ values, whose difference we want to correlate. Thus, we will have to:

For point [1] we will have $\frac{A U C}{A U C_{i}}=1+\frac{[I]_{\max }}{K_{i}}$; and for point [1'] we will have $\frac{A U C^{\prime}}{A U C_{i}}=1+\frac{[I]_{\max }^{\prime}}{K_{i}}$

The difference between them being equal to $\frac{A U C^{\prime}}{A U C_{i}}-\frac{A U C}{A U C_{i}}=\frac{[I]_{\max }}{K_{i}}-\frac{[I]_{\max }}{K_{i}}$ and, therefore, $\frac{\Delta A U C}{A U C_{i}}=\frac{\Delta[I]_{\max }}{K_{i}}$. For a $\Delta \approx 0$, we can write $\frac{d A U C}{A U C_{i}}=\frac{d[I]_{\max }}{K_{i}}$

Taking into account that $\mathrm{Ki}$ represents an [I], and integrating we will have:. $A+\operatorname{Ln} \frac{A U C}{A U C_{i}}=B+\operatorname{Ln} \frac{[I]_{\max }}{K_{i}}$. Finally replacing $[\mathrm{I}]$ max with TR we will have: 
$A+\operatorname{Ln}\left(A U C_{r}\right)=B+C\left(\operatorname{Ln} \frac{T R}{K_{i}}\right)$, Eq.2

Accordingly to Eq.2, we only analysed a log-log linear-regression models constructed on the basis of two main variables: the average of $\mathrm{AUCr}$ of the victim-drugs $\left(A U D r r_{a v g}\right)$ and the mean of the TR value-to- $K_{i}$ ratio $\left(\mathrm{TR}_{\mathrm{m}}-\mathrm{to}_{\mathrm{i}} \mathrm{K}_{\mathrm{i}}\right)$ of the inhibitors.

For this, we assumed the following criteria and conditions: dose -proportionality between the dose to achieve the maximum and the average concentrations of inhibitors at $\mathrm{SS}\left([\mathrm{I}]_{\max }\right.$ and $[\mathrm{I}]_{\mathrm{avg}}$, respectively); and, thus, since TR refers to either the dosage or the SS-plasma concentration interval at which the desired therapeutic effect is obtained $\left([\mathrm{I}]_{\mathrm{avg}}\right)$, TR can be used as a proxy of the $[\mathrm{I}] \max$.

Inclusion criteria: CYP2D6-mediated DDIs with available clinical data of AUCr, obtained from the literature, and victim drugs metabolized exclusively or mostly by CYP2D6. Exclusion criteria: DDIs whose inhibitor was time-dependent, the AUCr was $<1.1$ or TR data was not available.

Then, we collected data on in vivo AUCr and in vitro Ki, from 58 DDI studies involving CYP2D6 based on the dextromethorphan O-oxidation methodology, obtained from the literature review (data from Ito et al.). ${ }^{6}$ We also gathered bibliographic data on the TR for the 19 inhibitors involved in such DDIs (data from Regenthal et al.). ${ }^{7}$ After excluding, according exclusion criteria, the DDIs corresponding to omeprazole $(n=3)$, because it is not an inhibitor of CYP 2D6 (AUCr $<1.1)$, paroxetine $(n=7)$ because it is a time-dependent inhibitor of CYP2D6, ${ }^{8}$ and DDIs corresponding to ritonavir $(n=1)$ because the value of its therapeutic range was not available, the final sample to study was composed of 47 DDIs and 16 reversible inhibitors. These data are summarized in Table 1. 
Table 1. Values of inhibition constant $\left(K_{i}\right.$, in $\left.\mu M\right)$ and mean value of therapeutic range $\left(T R_{m}\right.$, in $\left.\mu M\right)$, calculated as the mean value between the maximum and minimum therapeutic range, of the inhibitors.

\begin{tabular}{|l|l|l|}
\hline \multicolumn{1}{|c|}{ Inhibitor } & $\mathbf{K}_{\mathbf{i}}$ & $\mathbf{T R}_{\mathbf{m}}$ \\
\hline Amiodarone & $11.8^{*}$ & 3 \\
\hline Amitriptyline & 30 & 0.43 \\
\hline Chlorpromazine & 4.8 & 0.28 \\
\hline Cimetidine & $7.7 *$ & 6.44 \\
\hline Citalopram & 24 & 0.32 \\
\hline Diltiazem & 150 & 0.42 \\
\hline Diphenhydramine & 10 & 0.25 \\
\hline Fluoxetine & 0.54 & 0.24 \\
\hline Fluvoxamine & $0.83^{*}$ & 0.39 \\
\hline Hydroxychloroquine & 66 & 0.89 \\
\hline Labetalol & 7 & 1.1 \\
\hline Mexiletine & 30 & 7.54 \\
\hline Propafenone & $0.03^{*}$ & 4.99 \\
\hline Quinidine & $0.02^{*}$ & 9.26 \\
\hline Sertraline & $0.47 *$ & 0.38 \\
\hline Verapamil & 16 & 0.33 \\
\hline
\end{tabular}

$K_{i}$ and AUCr data from Ito et al [Br J Clin Pharmacol 2004;57:473-86], ${ }^{6}$ except

${ }^{*} K_{i}$ from Van den Brink et al.[ Drug Metab Dispos 2012;40:47-53].

Finally, the correlation strength of the linear regression models was assessed by Pearson`s correlation coefficient.

\section{RESULTS}

As result, data were fitted to a linear log-log regression model, which obtained the best correlation between the following two variables: the AUCr of the victim-drug, calculated as the mean from the values of different DDIs between several victim-drugs with the same inhibitor ( $\mathrm{AUCr}_{\mathrm{avg}}$ ), and the mean therapeutic range of inhibitor-toinhibition constant ratio of the inhibitor $\left(\mathrm{TR}_{\mathrm{m}}-\mathrm{to}-\mathrm{K}_{\mathrm{i}}\right)$, calculated as the mean value between the maximum and minimum therapeutic range of the inhibitor divided by its inhibition constant $\left(\mathrm{TR}_{\mathrm{m}} / \mathrm{K}_{\mathrm{i}}\right)$. Data are summarized in Table 2 . 
Table 2. Cytochrome P450 2D6-mediated drug-drug interactions (DDI) studied: values of the area under curve ratio (AUCr) of the victim drugs; and values of the two correlated variables: $L n A U C r_{\text {avg }}=$ the neperian logarithm of the averaged AUCr of several victim drugs with the same inhibitory drug (see second column), and $\operatorname{Ln}\left(T R_{m}-\right.$ to- $\left.K_{i}\right)=$ neperian logarithm of the mean therapeutic range of inhibitorto-inhibition constant ratio.

\begin{tabular}{|c|c|c|c|}
\hline \multicolumn{2}{|r|}{ Drug-Drug Interaction } & \multirow[t]{2}{*}{$L n T R_{m}-t o-K_{i}$} & \multirow[t]{2}{*}{ Ln $A U C r_{a v g}$} \\
\hline Inhibitor & Victim Drug (AUCr) & & \\
\hline Amiodarone & FLC (1.37) & -1.4 & 0.31 \\
\hline Amitriptyline & ATN (1.24), MTP (1.44) & -4.2 & 0.29 \\
\hline Chlorpromazine & $\operatorname{PRPr}(1.69)$ & -2.8 & 0.52 \\
\hline Cimetidine & $\begin{array}{l}\text { ATN (1.07),MTP (1.61), PRPr(1.91; } \\
1.94 ; 1.47)\end{array}$ & -0.18 & 0.47 \\
\hline Citalopram & DSP (1.47), IMP (1.15), LVM (0.74) & -4.3 & 0.11 \\
\hline Diltiazem & $\begin{array}{l}\text { ATN (1.07),MTP (1.33),PRPr (1.48; } \\
1.33)\end{array}$ & -5.87 & 0.26 \\
\hline $\begin{array}{l}\text { Diphenhydramin } \\
\text { e }\end{array}$ & MTP $(1.61)$ & -3.66 & 0.48 \\
\hline Fluoxetine & $\begin{array}{l}\text { CRV (1.77), DSP (4.80; 7.43; 5.31), } \\
\text { IMP (3.33),PRPh 1.50), } \\
\text { RTV(1.19),TLT(4.84) }\end{array}$ & -0.81 & 1.33 \\
\hline Fluvoxamine & DSP (1.14), IMP (3.63) & -0.75 & 0.87 \\
\hline Hydroxychloroq & MTP (1.65) & -4.3 & 0.50 \\
\hline Labetalol & DSP (2.27), IMP (1.53) & -1.84 & 0.64 \\
\hline Mexiletine & MTP (1.82) & -1.38 & 0.60 \\
\hline Propafenone & $\operatorname{PRPr}(2.13)$ & 5.11 & 0.76 \\
\hline Quinidine & $\begin{array}{l}\text { DSP (7.5), ENC (3.18; 11.4), IMP } \\
\text { (1.54), MTP (3.24), MXL (1.32), } \\
\text { PRP (2.7) }\end{array}$ & 6.14 & 1.48 \\
\hline Sertraline & $\begin{array}{l}\operatorname{DSP}(1.2 ; 1.37 ; 1.74 ; 1.54 ; 2.29) \\
\operatorname{IMP}(1.68)\end{array}$ & -0.22 & 0.49 \\
\hline Verapamil & $\operatorname{MTP}(1.33), \operatorname{PRPr}(1.42)$ & -3.88 & 0.32 \\
\hline
\end{tabular}

ATN=Atenolol; $C R V=$ carvedilol; $D S P=$ desipramine; $E N C=$ encainide;

$F L C=$ flecainide; $I M P=$ imipramine; $L V M=$ levomepromazine; $M T P=$ metoprolol; $M X L=$ mexiletine; $P R P h=$ perphenazin $P R P=$ propafenone; $P R P r=$ propranolol; $R T V=$ Ritonavir $; T L T=$ tolterodine.

The equation for the linear regression obtained was: $\mathrm{Ln} \mathrm{AUCr}$ avg $=0.08 \mathrm{Ln}$ $\left(\mathrm{TR}_{\mathrm{m}}\right.$-to- $\left./ \mathrm{Ki}\right)+0.71 ;$ Eq.2, $\left(\mathrm{r}^{2}=0.72 ;\right.$ standard error $=0.069$, CI $95 \%$ : from 0.60 to 0.84 , $\mathrm{P}=0.0116$ ), where the slope measures the proportionality between two variables, $\mathrm{Ln}$ AUCr avg and $\mathrm{Ln}\left(\mathrm{TR}_{\mathrm{m}}-\mathrm{to}^{-} / \mathrm{K}_{\mathrm{i}}\right)$. The plot of this regression line is illustrated in: 
Fig. 1: Plot of regression line between the neperian logarithm of the mean of the therapeutic range-to-inhibition constant ratio of the inhibitory drug ( $L n T R{ }_{m}$-to- $K_{i} ; X$ independent variable) and the neperian logarithm of the average of the of area under the plasma concentration-time curve ratio of the victim drugs (Ln AUCr avg; Y-dependient variable).

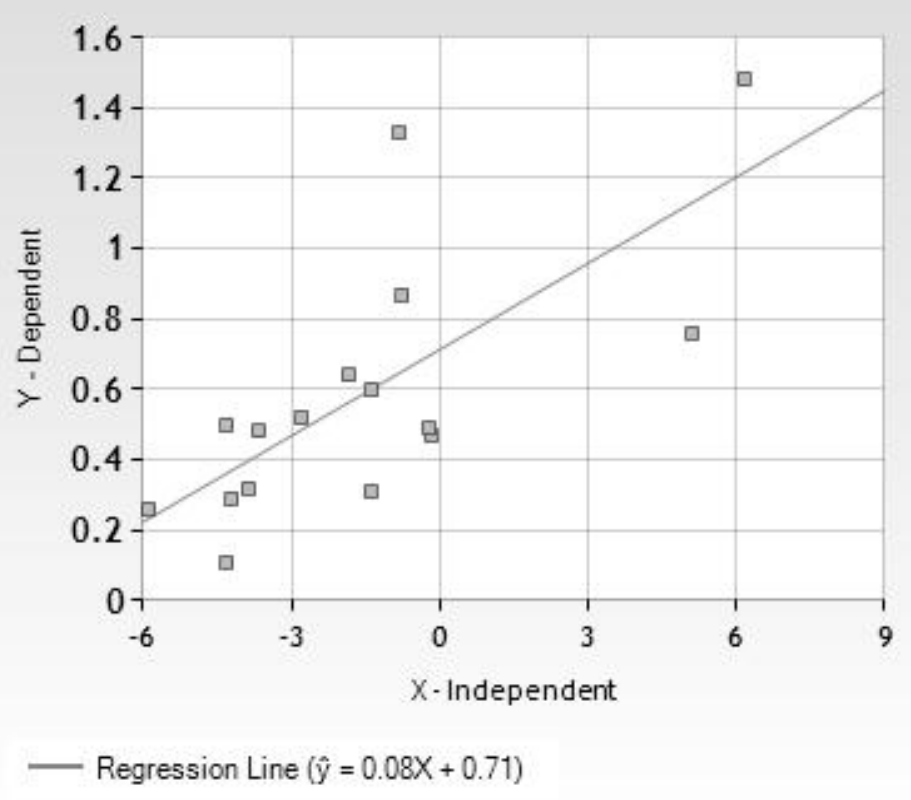

\section{DISCUSSION}

Due to the increase of the adverse events caused by the DDIs, the development and the application of new methods for the detection of them, constitutes one of the most important roles of the clinical pharmacologist.

This model shows good predictive results for a panel of 47 DDIs and 16 CYP2D6 inhibitors, constituting a valuable, quick, simple and extensible regressionbased method for the prediction of the intensity and clinical relevance of a metabolicbased DDIs mediated by CYP2D6 and their management in clinical practice, by using TR data of the inhibitors.

According to results, the simply knowledge of $\mathrm{K}_{\mathrm{i}}$ and TR of the inhibitor, would be sufficient to determine the intensity and clinical relevance of a DDI. In this sense, if $\mathrm{K}_{\mathrm{i}}$ exceeds the maximal value of $\mathrm{TR}$, then that drug is unlikely to inhibit the activity of 
that enzyme, and vice versa. The DDI management options would include adjusting dose of victim and/or inhibitor by applying data of TR and $\mathrm{K}_{\mathrm{i}}$ of inhibitor, from literature, to the equation of regression line presented here.

In fact, consistent with the correlated data and since the TR of an inhibitor refers both to the dosage range and to the plasma concentration at which the desired therapeutic effect is obtained, we can manage a DDI by dose adjustments of the CYP2D6 inhibitor, within the normal TR, in proportion to the magnitude of the desired change in the exposition to victim drug (AUCr), or directly by dose adjustments of victim drug aimed to avoid the appearance of ADRs. Also, would be useful to assign the probability that a particular drug causes a clinically relevant interaction, once the main routes of elimination of the interacting drugs and the potential factors that influence them have been previously identified.

Therefore, the present study constitutes a valid instrument for the management of the analysed CYP2D6-based DDIs, as well as an approach to make extrapolations in the case of DDIs for which no studies have been conducted, as would be the case the new interactions that arise when new drugs are incorporated, for which plasma concentration of inhibitors are not available for the prediction and management of a CYP2D6-mediated DDI. This would be much more important in the case of the victim drugs with a narrow margin of safety and for planning dosage regimens with a better benefit/risk ratio without increasing time and/or cost.

However, it is important to appreciate that the present analysis is empirical, and must be regarded as an initial step in the prediction of CYP2D6-based DDIs and further research will be needed to demonstrate its clinical applicability.

Finally, as weaknesses of the present study, it should be noted that factors such as the role of hepatic uptake transporters, the existence of more than one elimination 
pathway/metabolic pathway, the influence of multisite kinetics for CYP2D6, chirality of victim drugs and inhibitors, and nonlinear kinetics of substrates were not taken into account to achieve these results. These must be considered alongside the TR-to-Ki ratio to improve the prediction of CYP-mediated DDIs. In fact, the coefficient of determination $(\mathrm{R}=0.52)$, which explains the percentage of the total variation observed in the dependent variable, indicates that the regression line obtained can explain $52 \%$ of the total variation observed, while that most of the remaining $48 \%$ should be looked for by other factors.

\section{CONCLUSIONS}

A drug-drug interaction involving reversible inhibitory drugs of CYP2D6 could be manage by adjusting dose of victim and/or inhibitory drug, according to the magnitude of the desired change, by applying data of therapeutic range and inhibition constant of inhibitor from literature to the equation of regression line presented here.

\section{ETHICS APPROVAL AND CONSENT TO PARTICIPATE}

Not applicable.

\section{CONSENT TO PUBLISH}

Not applicable.

\section{FUNDING}

The authors received no specific funding for this work.

\section{AUTHORS' CONTRIBUTIONS}

All authors participated in research design, data analysis and the writing of the manuscript. All authors approve the final version of the manuscript.

\section{CONFLICTS OF INTEREST}

The authors declare that they have no conflicts of interest.

\section{REFERENCES}


1.Lozano, R. and Frutos, A. J. 2018, Int. J. Clin. Pharm. Ther., 56,28-30.

2.https://www.fda.gov/downloads/Drugs/GuidanceComplianceRegulatoryInformation/ Guidances/UCM581965.pdf (Accessed 3.10. 2018).

3. Fowler, S. and Zhang, H. 2008, A.A.P.S. J., 10(2), 410-24.

4.Cheng, Y. and Prusoff, W. H. 1973, Biochem. Pharmacol., 22(23), 3099-108.

5. Zhang, Z. Y. and Wong,Y. N. 2005, Curr.Drug Metab., 6, 241-57.

6. Ito, K., Brown, H. S. and Houston, J. B. 2004, Br. J. Clin. Pharmacol., 57, 473-86.

7. Regenthal, R., Krueger, M., Koeppel, C. and Preiss, R. 1999, J. Clin. Monit. Comput., 15, 529-44.

8. Bertelsen, K. M., Venkatakrishnan, K., Von Moltke, L. L., Obach, R. S. and Greenblatt, D. J. 2003, Drug Metab. Dispos., 31, 289-93.

9.Van den Brink, B. M., Foti, R. S., Rock, D. A., Wienkers, L. C. and Wahlstrom, J. L. 2012, Drug Metab. Dispos., 40, 47-53. 